\title{
Identification and validation of an immune- associated RNA-binding proteins signature to predict clinical outcomes and therapeutic responses in colon cancer patients
}

\author{
Di Sun ${ }^{1}$, Kui-Sheng Yang ${ }^{2}$, Jian-Liang Chen ${ }^{2}$ and Zheng-bing Wang ${ }^{*^{*}}$ (D)
}

\begin{abstract}
Background: The immune infiltration of patients with colon cancer (CC) is closely associated with RNA-binding proteins (RBPs). However, immune-associated RBPs (IARBPs) in CC remain unexplored.

Methods: The data were downloaded from The Cancer Genome Atlas (TCGA) and the patients were divided into four immune subgroups by single sample gene set enrichment analysis (sSGSEA), in which weighted gene correlation network analysis (WGCNA) identified modules of co-expressed genes correlated with immune infiltration. Univariate (UCR) and multivariate Cox regression (MCR) analyses were applied to screen survival-associated IARBPs. Then, a prognostic signature was performed on TCGA dataset. Risk model was constructed based on the TCGA dataset. Based on the median risk score, CC patients were subdivided into low- and high-risk groups. Furthermore, the accuracy and prognostic value of this signature were validated by using Kaplan-Meier (K-M) curve, receiver operating characteristic (ROC). We further validated the findings in Gene Expression Omnibus (GEO) database. Finally, we evaluated the association between gene expression level and drug sensitivity.

Results: Based on the infiltration of immune cells, the TCGA patients were divided into four subgroups. In total, we identified 25 IARBPs, after differential expression and WGCNA analysis. Subsequently, two IARBP signatures (FBXO17 and PPARGC1A) were identified to be significantly associated with the overall survival (OS) of CC patients. K-M survival analysis revealed that the low-risk group correlated with prolonged OS. The prognostic signature was an independent prognostic factor and reflects the immune status of CC patients. Finally, FBXO17 was related with drug sensitivity of bleomycin, gemcitabine, and lenvatinib. PPARGC1A was related to drug sensitivity of dabrafenib, vemurafenib, and trametinib.
\end{abstract}

Conclusion: A novel two immune-associated RBPs that was established that may be useful in predicting survival and individualized treatment.

Keywords: Colon cancer, RNA-binding protein, Immune microenvironment, Prognosis model

*Correspondence: wzhb403@163.com

1 Department of Gastrointestinal Surgery, Affiliated Hospital of Yangzhou University, Yangzhou 225100, People's Republic of China

Full list of author information is available at the end of the article

\section{Introduction}

Colon cancer $(\mathrm{CC})$, one of the most common digestive malignant tumors, has become an important public health issue. Its ranks fourth in incidence and second as a cause of mortality among 36 cancer types worldwide [1]. The incidence of CC is high incidence original author(s) and the source, provide a link to the Creative Commons licence, and indicate if changes were made. The images or other third party material in this article are included in the article's Creative Commons licence, unless indicated otherwise in a credit line to the material. If material is not included in the article's Creative Commons licence and your intended use is not permitted by statutory regulation or exceeds the permitted use, you will need to obtain permission directly from the copyright holder. To view a copy of this licence, visit http://creativecommons.org/licenses/by/4.0/. The Creative Commons Public Domain Dedication waiver (http://creativeco mmons.org/publicdomain/zero/1.0/) applies to the data made available in this article, unless otherwise stated in a credit line to the data. 
in the Western world and developed Asian countries [2]. Early detection and treatment have decreased morbidity and mortality. Numerous biomarkers have been revealed in CC [3, 4]. Unfortunately, recurrence or metastasis will occur in approximately $30-50 \%$ of patients within 5 years after treatment [5]. The effective prediction model can play a significant role for the accurate assessment of patients' prognosis and to optimize clinical treatment strategies [6-10]. Recently, a growing number of evidences indicate that the potential effect of the tumor immune microenvironment (TIM) have great potential for predicting the efficacy of immunotherapy as well as prognosis [11]. TIM is composed by immune cells. Adaptive anti-tumor immune responses have been correlated with tumor progression in various cancers, including $\mathrm{CC}$, and tumor development is also dependent on the infiltration of immune cells [12].

RNA-binding protein (RBP) plays a significant role in tumor progression via post-transcriptional modification [13]. Specifically, RNA-binding proteins (RBPs) play essential roles in RNA life, including pre-mRNA processing, modification, localization, RNA stability, and translation [14]. The inflammatory response has been reported to be modulated by modulating mRNA pools in both immune and nonimmune cells [15]. Considering that RBPs can regulate the infiltration degrees of immune cells, whether there are immune-associated RBPs that can be used to accurately evaluate the tumor progression and prognosis of $\mathrm{CC}$ patients has not yet been considered.

In the present study, we evaluated the association between the expression of immune-associated RBPs (IARBPs) and prognosis in CC patients. To this end, we train a model to predict the OS of patients with CC.

\section{Materials and methods Data processing}

The RNA-Seq data and clinical information of male CC patients were obtained from The Cancer Genome Atlas (TCGA) (398 tumor samples and 39 normal samples) and Gene Expression Omnibus (GEO) (GSE40967) database. The gene expression profile of 580 CC (GSE40967) based on the GPL570 (Affymetrix Human Genome U133 Plus 2.0 Array) platform was also downloaded. A total of 1542 RBPs were obtained from a previous study [16].

\section{Identification of CC subtypes based on ssGSEA score and differentially expressed genes identification}

For each CC dataset, 27 immune cell types were determined using the single sample gene set enrichment analysis (ssGSEA) software implemented in the R GSVA package. Consensus clustering was performed using the "ConsensusClusterPlus" package in $\mathrm{R}$ to identify subgroups based on ssGSEA scores. In brief, $k$-means clustering was used, with 50 iterations (each using $80 \%$ of the samples). The best-fit number of clusters was determined by the cumulative distribution function (CDF) curve and the changes in the area under the CDF curve. The stromal score, immune score, tumor purity, and estimate score of each included sample was calculated by ESTIMATE algorithm [17]. The CIBERSORT deconvolution algorithm (https://cibersort.stanford.edu/ assessed on 28 December 2020) was used to verify that the infiltration of immune cells from these four immune subtypes were different [18]. Differentially expressed genes (DEGs) between Cluster1 and Cluster2 were determined using the $\mathrm{R}$ package Limma. Genes with $P<0.05$, and [logFoldChange (logFC)] $>1$ were considered DEGs. Using the gplots and heatmap in the edgeR package, volcano plots and heat maps of DEGs were constructed.

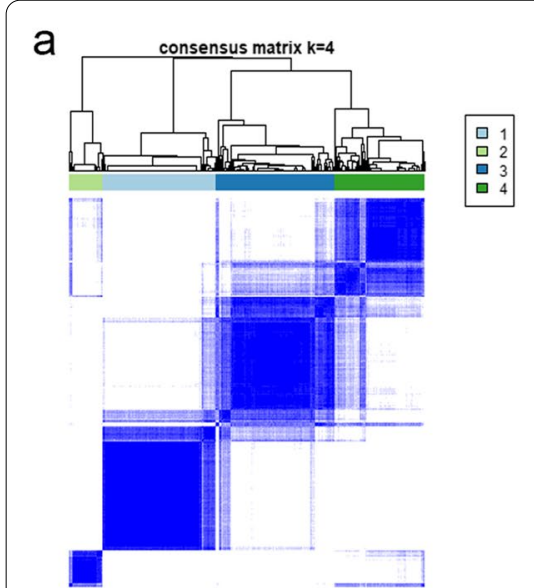

b

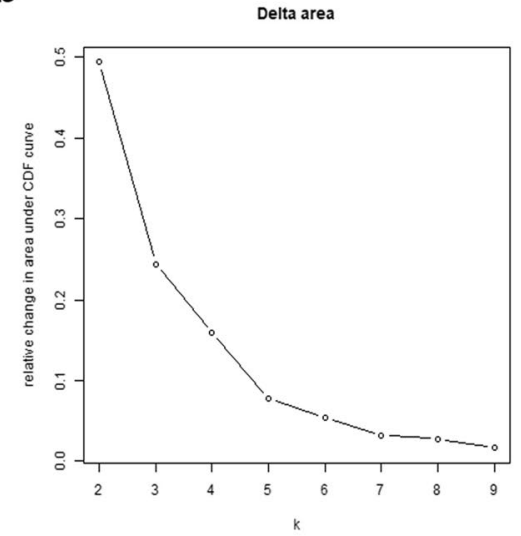

C

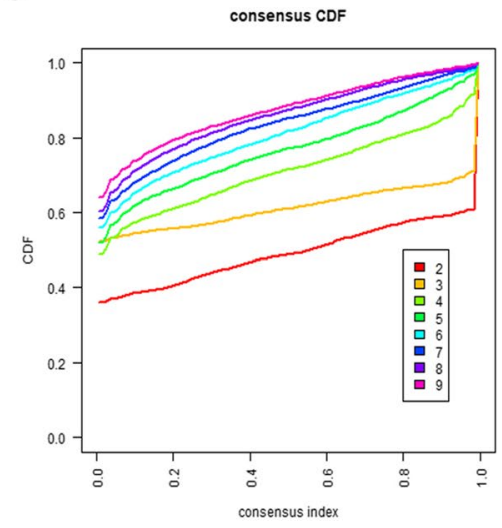

Fig. 1 Consensus clustering of colon cancer (CC). a The TCGA samples were divided into four distinct clusters when $k=4$. $\mathbf{b}$ Relative change in area under cumulative distribution function (CDF) curve for $k=2-9$. c Consensus clustering CDF curve for $k=2-9$ 


\section{Weighted gene co-expression network analysis}

DEGs were used to construct a weight co-expression network using the R package "WGCNA" [19]. The topological overlap measure (TOM) was clustered, and gene modules were identified. We calculated the module eigengene (ME) of each module, which represents the expression level for each module. The threshold was set as $P<0.05$.

\section{Function and pathway analysis}

Gene Ontology enrichment analyses of module genes performed using the Metascape (https://metascape.org/), a gene annotation and analysis resource.

\section{Building a prognostic model}

Univariate Cox regression (UCR) analysis was performed to screen out which RBP-related genes associated with the OS of patients. Then, the multivariate Cox regression (MCR) test for the coefficients (bi) of the hub genes was performed. This model was developed as Risk score $=\sum \mathrm{i}$ $=1 \mathrm{~N}(\operatorname{Exp}(\mathrm{i}) \cdot \operatorname{coe}(\mathrm{i}))$. Based on the median risk score, the patients were divided into low-risk or high-risk groups. Then, the ROC curve was conducted to evaluate the predictive accuracy. The $\mathrm{K}-\mathrm{M}$ survival curve was done to assess whether there was a survival difference between 2 categories. To validate the result, we implemented the same procedure on the validation cohort.

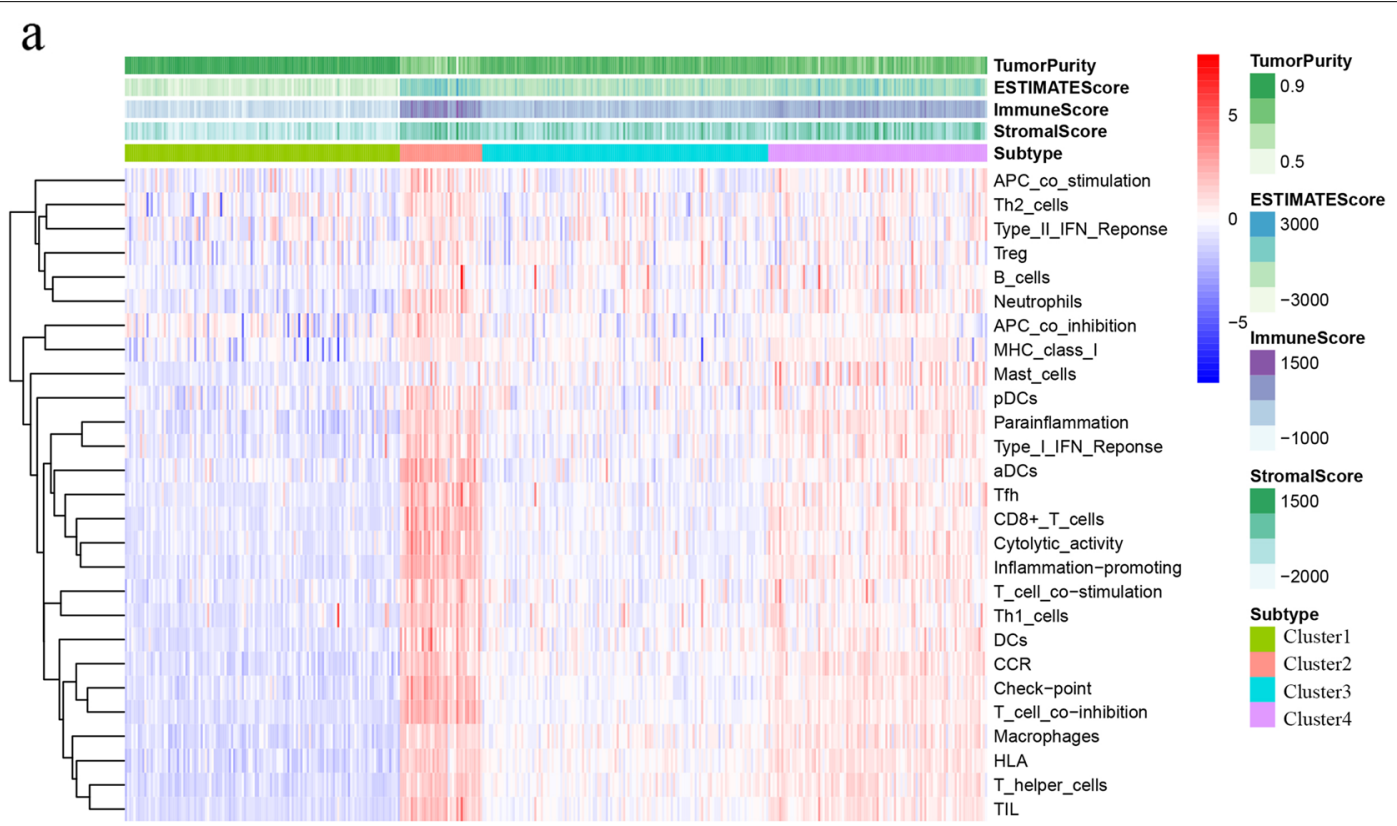

b

c
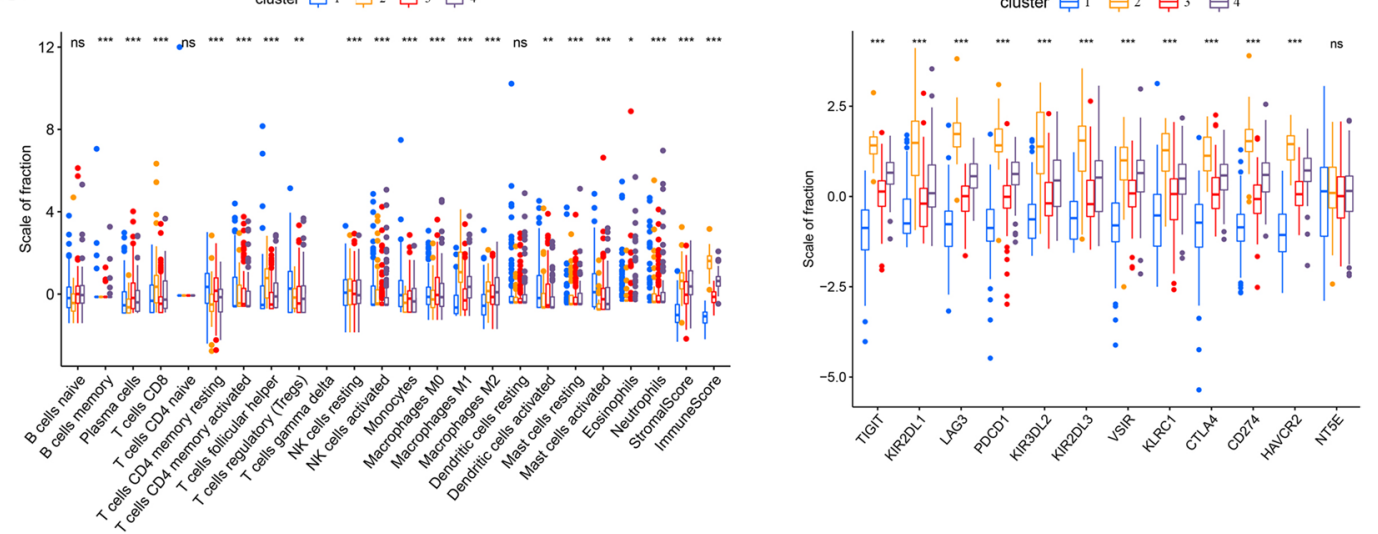

Fig. 2 Determination of four immune subtypes in the CC. a Heatmap of ssGSEA scores. $\mathbf{b}$ The differences in the infiltrating immune cells between four clusters. $\mathbf{c}$ The differences in the expressions of immune checkpoint genes consisting among four subtypes. ${ }^{*} P<0.05$, ${ }^{* *} P<0.01,{ }^{* * *} P<0.005$ 

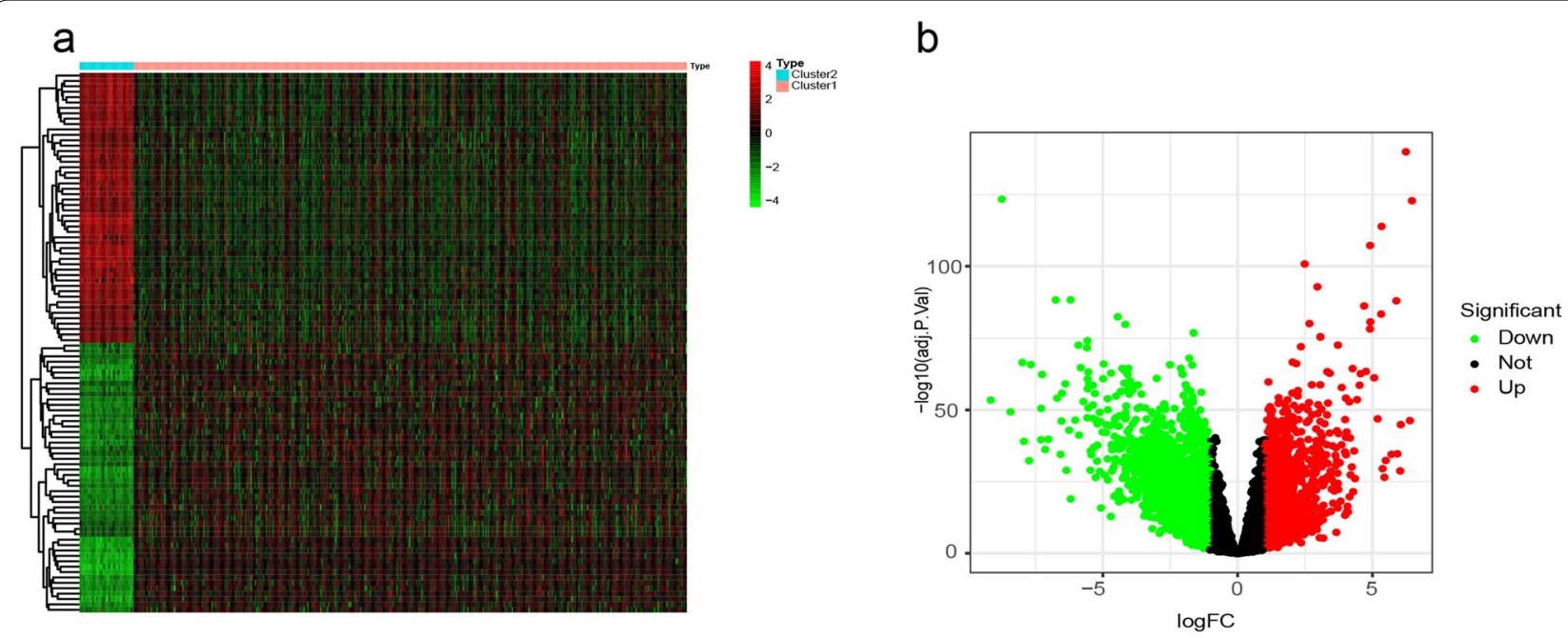

Fig. 3 Analysis of differentially expressed genes. a Heatmap of differentially expressed genes. $\mathbf{b}$ The volcano graph shows the distribution of differential genes between Cluster1 and Cluster2, the red and blue dots represent significantly up- and downregulated expressed genes, respectively

a

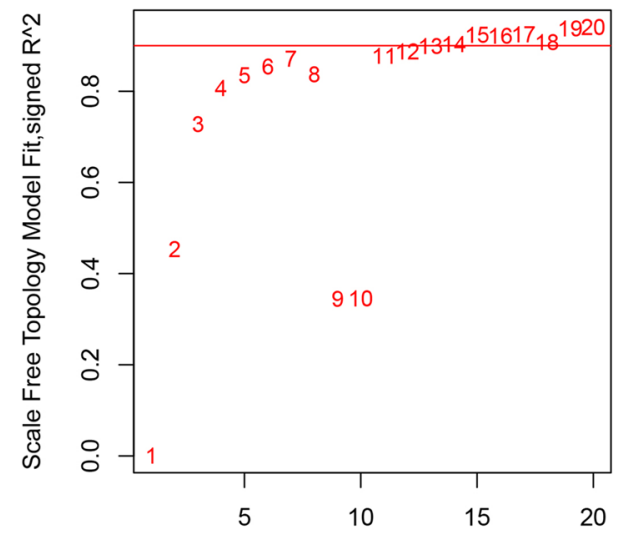

b

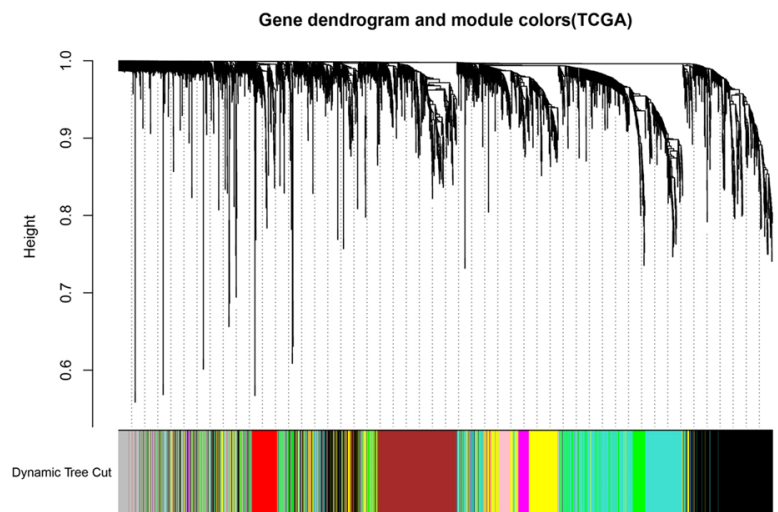

Mean connectivity

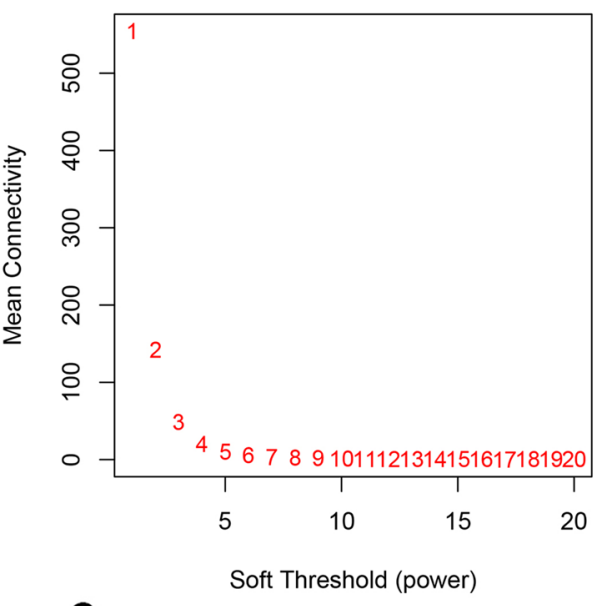

C

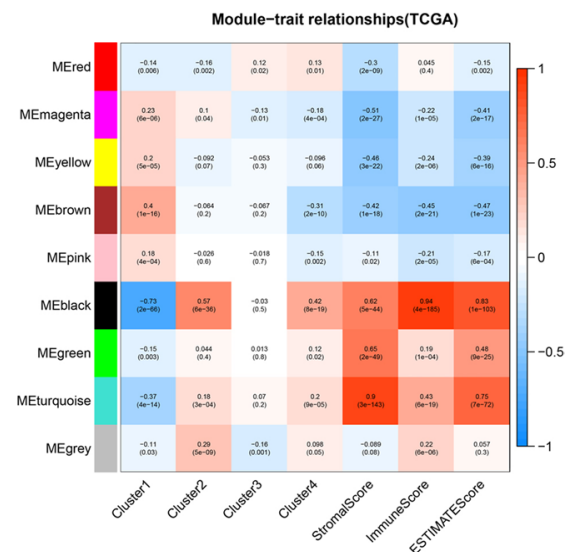

Fig. 4 Weighted co-expression gene network. a Determination of the $\beta$ parameter value in the adjacency function. $\mathbf{b}$ Nine gene modules were merged 


\section{Drug sensitivity correlation analysis}

The drug sensitivity was downloaded from the CellMiner database (https://discover.nci.nih.gov/cellminer/) [20, 21]. R package "impute", "limma", "ggplot2", and "ggpubr" were used for the data processing, statistical analysis, and result visualization.

\section{Statistical analysis}

Overall survival was estimated by $\mathrm{K}-\mathrm{M}$ analysis with log-rank test. The Wilcoxon rank-sum test was used to compare these measures between the groups. To compare three or more groups, a Kruskal-Wallis test was used. Correlation (Pearson correlation coefficient $r$ ) assessed strength and direction of the linear relationship between two variables. Statistical analyses and data visualization were performed in R programing language. $P$-values $(p)<0.05$ were considered statistically significant.

\section{Results}

Identification of CC immune subtypes based on infiltration of immune cells

Based on ssGSEA scores, the TCGA CC samples in this data set have been clustered into four immune infiltration subtypes: 127 cases in Cluster 1,38 cases in Cluster1, 132 cases in Cluster3, and 101 cases in Cluster4 (Figs. 1 and 2a). The CC cases with higher immune infiltration had higher ESTIMATE, stromal, and immune scores but a lower tumor purity among the 22 types of immune cells $(P<0.05)$. The infiltration of various types of immune cells showed a progressive increasing or decreasing trend, such as CD8 T cells, T cells follicular helper, NK cells resting, macrophages M1, and macrophages M2 have differences infiltration degree in different subtypes $(P<0.001)$ (Fig. 2b). Finally, the expression profiles of 12 immune checkpoint genes (ICIs) (i.e., LAG3, VSIR, CD274, KIR2DL1, KLRC1, HAVCR2, NT5E CTLA4, TIGIT, PDCD1, KIR3DL2, and KIR2DL3), which are crucial for immune modulation, were further examined. The expression correlated with high levels of immune infiltration cluster (Fig. 2c).

\section{Identification of DEGs}

In the TCGA dataset, we identified 3572 DEGs between the high- and low-infiltration subgroup (Cluster1 VS Cluster2) with the criteria of $P<0.05$ and $[\operatorname{logFC}]>1$. Of the 3572 genes, 1343 genes were upregulated and 2229 downregulated. An expression volcano plots and heat map were indicated in Fig. 3a and b.

\section{Identification of co-expression modules}

We employed the WGCNA to analyze the DEGs. A soft threshold (power $=7$ ) was selected by standard scale-free model fitting index $\mathrm{R} 2=0.92$ (Fig. 4a). Three modules shown in turquoise, green, and black in Fig. 4c were positively correlated with Cluster2,

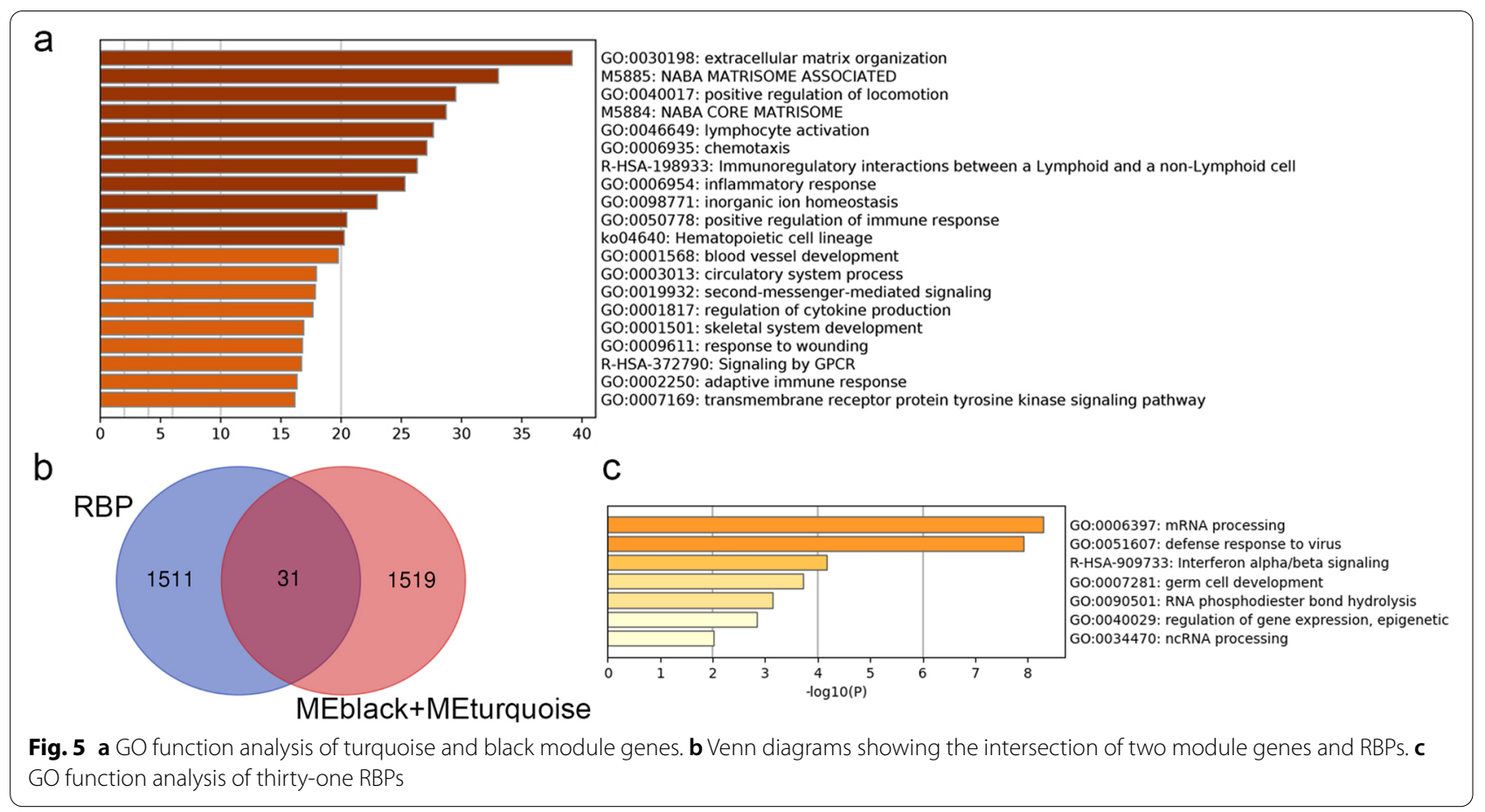


Cluster4, Stromal score, Immune score, and ESTIMATE score. Three modules shown in magenta, yellow, and brown negatively correlated with Cluster2, Cluster4, Stromal score, Immune score, and ESTIMATE score (Fig. 4c). Next, turquoise and black module were chosen to further analysis. Total 1550 genes from the two modules were applied to Gene Ontology (GO) analysis. The GO terms lymphocyte activation, immunoregulatory interactions between a lymphoid and a non-Lymphoid cell, inflammatory response, and positive regulation of immune response were the enriched GO terms (Fig. 5a). Then, we obtained 31 RBPs by taking the intersection of turquoise and black module genes and 1542 RBPs (Fig. 5b; Table 1). $\mathrm{GO}$ analysis revealed that the majority of these genes were functionally related to Mrna processing, defense response to virus, and interferon alpha/beta signaling (Fig. 5c; Table 2).

\section{Construction of prognostic signatures based on RBP}

From UCR analysis, we identified two candidate RBP genes associated with the prognosis in patients with CC (FBXO17 and PPARGC1A) (Fig. 6). Based on these findings, we established a $\mathrm{CC}$ prediction model by MCR analysis. We calculated the risk score for each patient by the following formula: Risk score $=(0.1707 \times \operatorname{Exp}[$ FBXO17] $)+(-$ $0.1515 \times \operatorname{Exp}$ [PPARGC1A]). Patients in the TCGA set were divided into high- or low-risk groups with the median risk score. K-M survival curves showed the OS was significantly worse in the highrisk group compared with the low-risk group $(P=$ $6.518 \mathrm{e}-03$ ) (Fig. 7a). From the scatterplot and risk curve, the survival status of patients with different risk scores; the mortality rate remarkably increased with the higher risk score (Fig. 7b, c). Heatmap of expression profiles in TCGA indicated that FBXO17 were highly expressed in the high-risk group, but PPARGC1A were highly expressed in the low-risk group (Fig. $7 \mathrm{~d}$ ). Besides, the area under the ROC (AUC) values were 0.733 (Fig. 8a), showing a good capacity of two RBP genes in predicting overall survival. The prognostic significance of the prognostic mode was further validated by the validation cohort (Figs. 7 and 8 e, $h$ and d).

\section{Independent prognostic value of the risk model}

We performed UCR analysis revealed that higher risk scores had shorter OS (HR, 1.984; 95\% CI, 1.280-3.075; $P=0.002$ ) (Fig. 8b). Several clinicopathologic variables displayed a relationship with prognosis included age, stage, metastasis, $\mathrm{T}$, and $\mathrm{N}$. Additional MCR analysis indicated that analyses indicated that
Table 1 The specific information of 31 RBPs

\begin{tabular}{ll}
\hline Gene Symbol & Description \\
\hline SIDT1 & SID1 transmembrane family member 1 \\
A1CF & APOBEC1 complementation factor \\
IPO4 & importin 4 \\
DNMT3B & DNA methyltransferase 3 beta \\
QKI & QKI, KH domain containing RNA binding \\
PPARGC1A & PPARG coactivator 1 alpha \\
MEX3A & mex-3 RNA binding family member A \\
DZIP1 & DAZ interacting zinc finger protein 1 \\
TLR7 & toll like receptor 7 \\
RNASE1 & ribonuclease A family member 1, pancreatic \\
POLR1G & RNA polymerase I subunit G \\
RBFOX3 & RNA binding fox-1 homolog 3 \\
DDX27 & DEAD-box helicase 27 \\
RBM24 & RNA binding motif protein 24 \\
PABPC3 & poly(A) binding protein cytoplasmic 3 \\
ZCCHC24 & zinc finger CCHC-type containing 24 \\
IFIT2 & interferon induced protein with tetratricopeptide \\
TLR8 & repeats 2 \\
DDX60 & toll like receptor 8 \\
NPM2 & DExD/H-box helicase 60 \\
FBXO17 & nucleophosmin/nucleoplasmin 2 \\
ZC3HAV1L & F-box protein 17 \\
DQX1 & zinc finger CCCH-type containing, antiviral 1 like \\
CELF3 & DEAQ-box RNA dependent ATPase 1 \\
OASL & CUGBP Elav-like family member 3 \\
CDK5RAP1 & rep'-oligoadenylate synthetase like \\
PABPC1L & CDK5 regulatory subunit associated protein 1 \\
TSEN2 & poly(A) binding protein cytoplasmic 1 like \\
IFIT1 & \\
AZGP1 & RAVER2
\end{tabular}

high-level risk score served as an independent prognostic factor for poor survival in CC patients (HR, 2.167; CI, 1.366-2.346, $P=0.001$ ) (Fig. 8c). To determine whether the clinical prognostic model was reliable, we then utilized this same risk score formula to analyze patients in the GEO cohort, which was consistent with those found in the TCGA database (Fig. 8e, f).

\section{Immune profile in risk groups}

We used the CIBERSORT algorithm to assess the composition of the immune microenvironment and further reveal the differences of immune cell infiltration between the two risk groups. As shown in Fig. 9a, plasma cells, T cells CD4 memory resting, monocytes, and dendritic cells activated cells were downregulated 
in the high-risk group, while Macrophages M0, Macrophages M2, Stromal score, and Immune score were significantly upregulated $(P<0.05)$ (Fig. 9a). Furthermore, PPARGC1A and FBXO17 expression negatively or positively associated with the immune gene sets, respectively (Fig. 9b).

\section{Drug sensitivity analysis of FBXO17 and PPARGC1A}

The CellMiner database was exploited to analyze the relationship between the drug sensitivity and the expression of FBXO17 and PPARGC1A. Pearson's correlation analysis indicated that FBXO17 expression was negatively associated with Entinostat, LDK-378, Perifosine, PX-31, and Palbociclib drug sensitivity, and positively related to Bleomycin, Gemcitabine, Irofulven, Sonidegib, and Lenvatinib drug sensitivity. PPARGC1A expression was negatively related to Triciribine phosphate, 7-Hydroxystaurosporine, and Dasatinib drug sensitivity. PPARGC1A expression was positively related to Hydrastinine $\mathrm{HCl}$, Vemurafenib, and Dabrafenib drug sensitivity (Fig. 10).

Table 2 The biological processes of 31 RNA-binding proteins

\begin{tabular}{|c|c|c|}
\hline Category & Term & Description \\
\hline GO Biological Processes & GO:0006397 & mRNA processing \\
\hline GO Biological Processes & GO:0006397 & mRNA processing \\
\hline GO Biological Processes & GO:0008380 & RNA splicing \\
\hline GO Biological Processes & GO:0048024 & regulation of mRNA splicing, via spliceosome \\
\hline GO Biological Processes & GO:0050684 & regulation of mRNA processing \\
\hline GO Biological Processes & GO:0043484 & regulation of RNA splicing \\
\hline GO Biological Processes & GO:0000381 & regulation of alternative mRNA splicing, via spliceosome \\
\hline GO Biological Processes & GO:0000377 & $\begin{array}{l}\text { RNA splicing, via transesterification reactions with bulged adeno- } \\
\text { sine as nucleophile }\end{array}$ \\
\hline GO Biological Processes & GO:0000398 & mRNA splicing, via spliceosome \\
\hline GO Biological Processes & GO:0000375 & RNA splicing, via transesterification reactions \\
\hline GO Biological Processes & GO:0000380 & alternative mRNA splicing, via spliceosome \\
\hline GO Biological Processes & GO:1903311 & regulation of mRNA metabolic process \\
\hline GO Biological Processes & GO:0051607 & defense response to virus \\
\hline GO Biological Processes & GO:0051607 & defense response to virus \\
\hline GO Biological Processes & GO:0140546 & defense response to symbiont \\
\hline GO Biological Processes & GO:0009615 & response to virus \\
\hline GO Biological Processes & GO:0002221 & pattern recognition receptor signaling pathway \\
\hline Reactome Gene Sets & R-HSA-909733 & Interferon alpha/beta signaling \\
\hline Reactome Gene Sets & R-HSA-909733 & Interferon alpha/beta signaling \\
\hline GO Biological Processes & GO:0060337 & type I interferon signaling pathway \\
\hline GO Biological Processes & GO:0071357 & cellular response to type I interferon \\
\hline GO Biological Processes & GO:0034340 & response to type I interferon \\
\hline GO Biological Processes & GO:0043393 & regulation of protein binding \\
\hline Reactome Gene Sets & R-HSA-913531 & Interferon Signaling \\
\hline GO Biological Processes & GO:0051098 & regulation of binding \\
\hline GO Biological Processes & GO:0007281 & germ cell development \\
\hline GO Biological Processes & GO:0007281 & germ cell development \\
\hline GO Biological Processes & GO:0022412 & cellular process involved in reproduction in multicellular organism \\
\hline GO Biological Processes & GO:0010638 & positive regulation of organelle organization \\
\hline GO Biological Processes & GO:0007276 & gamete generation \\
\hline GO Biological Processes & GO:0090501 & RNA phosphodiester bond hydrolysis \\
\hline GO Biological Processes & GO:0090501 & RNA phosphodiester bond hydrolysis \\
\hline GO Biological Processes & GO:0090305 & nucleic acid phosphodiester bond hydrolysis \\
\hline GO Biological Processes & GO:0040029 & regulation of gene expression, epigenetic \\
\hline GO Biological Processes & GO:0040029 & regulation of gene expression, epigenetic \\
\hline GO Biological Processes & GO:0034470 & ncRNA processing \\
\hline GO Biological Processes & GO:0034470 & ncRNA processing \\
\hline
\end{tabular}




\section{Discussion}

$\mathrm{CC}$ is one of the devastating malignancies with poor prognosis [22, 23]. Screening for candidate biomarkers remains a great challenge in improving the prognosis and evaluating the therapeutic effect in future. However, at present, no robust and effective biological markers can accurately predict the survival and prognosis of CC patients. It is well-accepted that immunity also plays critical roles in cancer development and progression [24]. Immunotherapy is showing impressive success in cancer treatment $[25,26]$. Thus, it is significantly important to construct an immune-related prediction model, thus guiding the clinical treatment strategy.

Herein, CC patients were separated into four clusters based on the immune signature score. There are notable differences in tumor purity, ESTIMATE, stromal scores, immune scores, and the expression of immune checkpoint markers between the four clusters. The Cluster2 patients have a highest immune infiltration, and Cluster 1 have a lowest immune infiltration. WGCNA analysis found turquoise and black module with highly relevant expression pattern. Then, the identified module genes, we performed Gene Ontology (GO) enrichment analysis in biological processes for "turquoise" and "black" module, to explore the biological process of each module. Two modules including 1550 DEGs were involved in lymphocyte activation, immunoregulatory interactions between a lymphoid and a non-lymphoid cell, inflammatory response, and positive regulation of immune response. Many studies have confirmed that there is a close relationship between tumor immune and CC [27]. Thirty-one IARBPs in two modules were selected out for further screening, analysis, and construction of a prognostic signature composed of two IARBPs.

Canonical RBPs work by binding to conserved sequence motifs in their target mRNAs via combinations of structurally well-defined RNA-binding

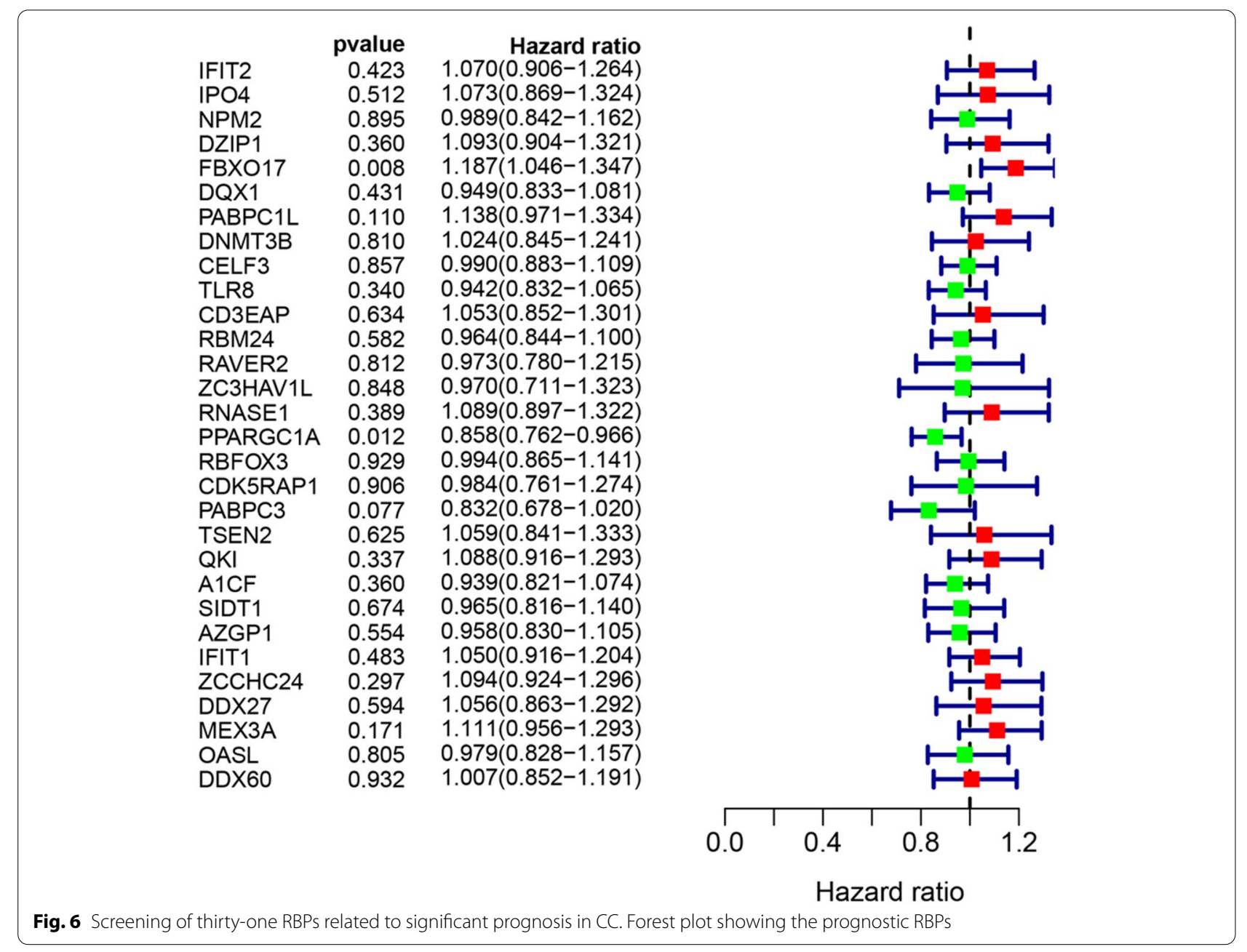


domains (RBDs) [28]. Classic RBDs include the RNA recognition motif, the K-homology, DEAD/DEAH helicase, and zinc-finger domains [16]. Besides, noncanonical RBPs refer to those proteins which have not been proved to have classic RBDs or the established domains by direct experimental evidence but have RNA-binding activity. In our prognostic signature, the FBXO17 and PPARGC1A are classic RBPs. FBXO17 primarily plays an oncogenic role in various cancers $[29,30]$. Over the past few years, studies have increasingly documented the contribution of FBXO17 to immune. For instance, FBXO17 inhibited activation of IFN-I signaling induced by various innate stimuli. Moreover, knockdown or knockout of FBXO17 promoted the transcriptional induction of IFN- $\beta$ and IFN-stimulated genes as well as antiviral activities [31]. PPARGC1A is a known master regulator of mitochondrial biogenesis [32]. PPARGC1A variant appears to be associated with the risk of CC [33]. Additionally, PPARGC1A may directly affect expression of genes with either pro- or anti-inflammatory functions [34]. However, there are few studies on the role of

\section{a}

a

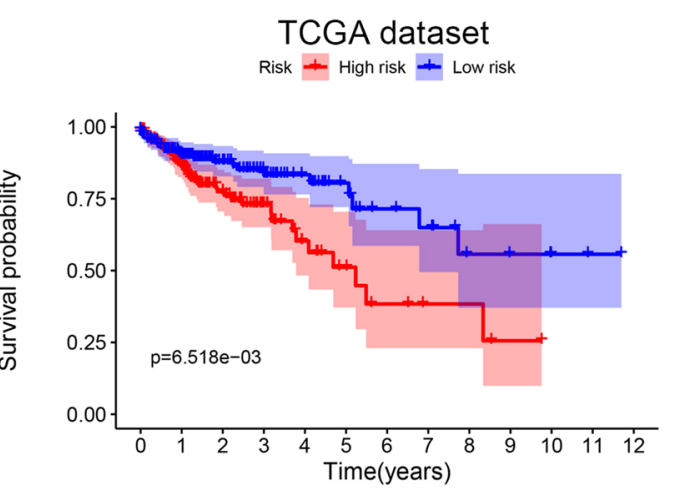

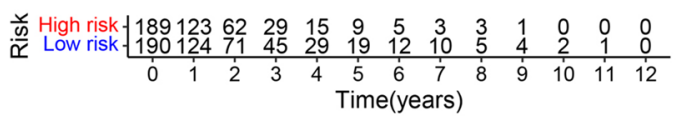

b

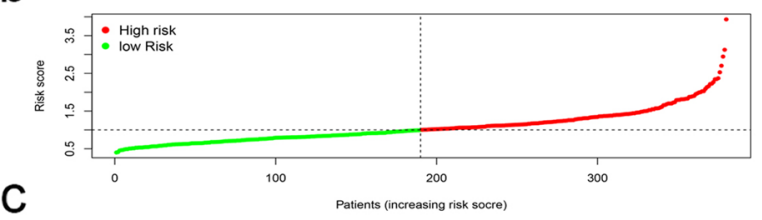

C

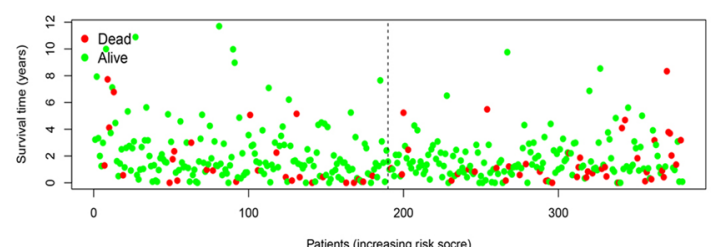

d

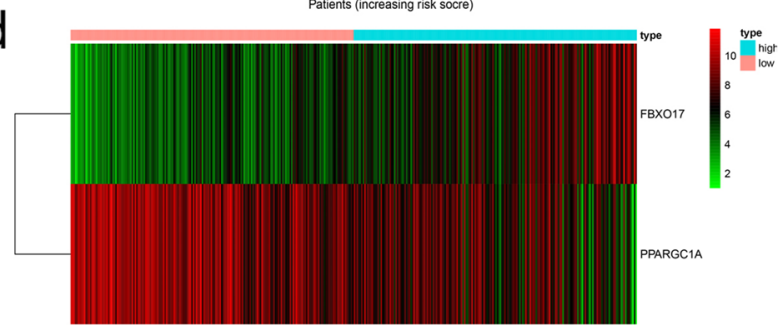

e

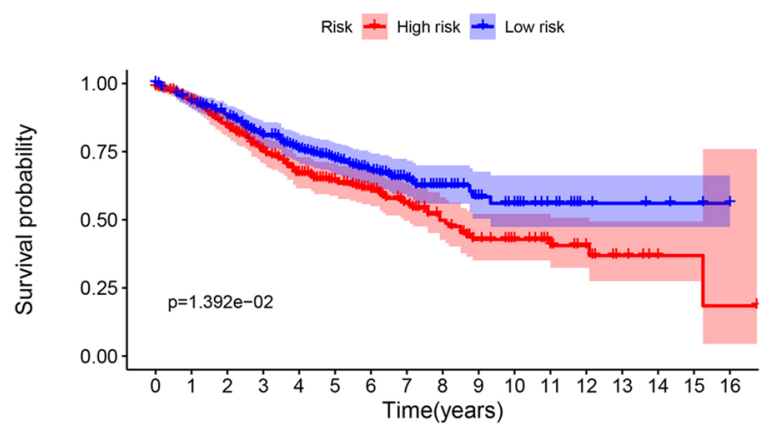

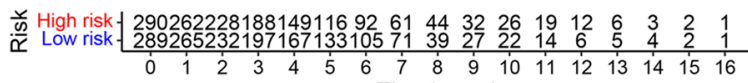
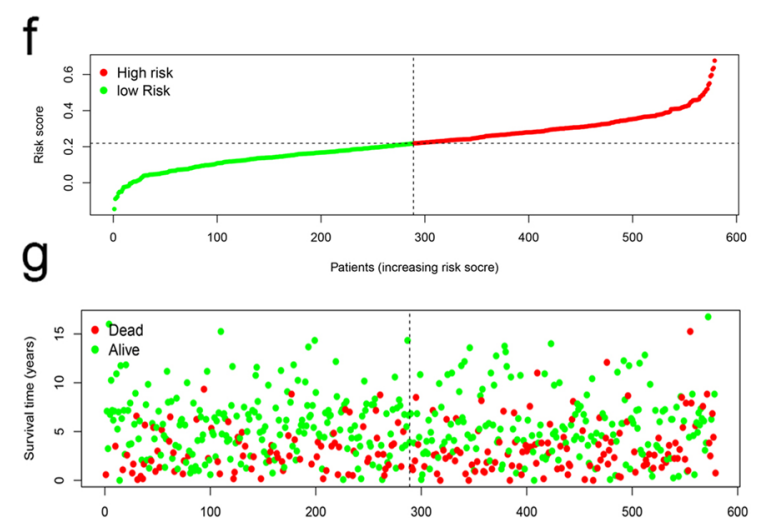

$\mathrm{h}$

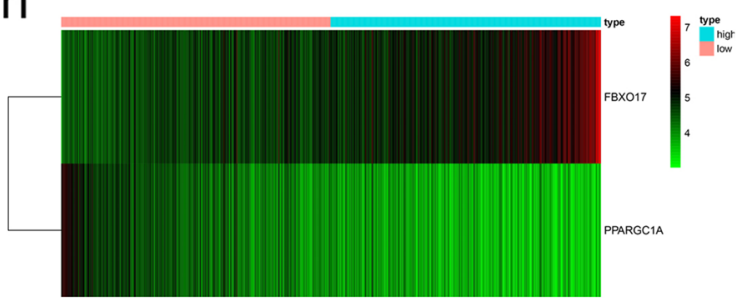

Fig. 7 The RBP signature comprising two genes, which predicted the overall survival of CC, in the TCGA and GEO cohorts. a K-M survival analysis, b risk score distribution, c survival status, and $\mathbf{d}$ heatmap of a prognostic model in the CC cohort from TCGA. e K-M survival analysis, $\mathbf{f}$ risk score distribution, $\mathbf{g}$ survival status, and $\mathbf{h}$ heatmap of a prognostic model in the CC cohort from GEO 


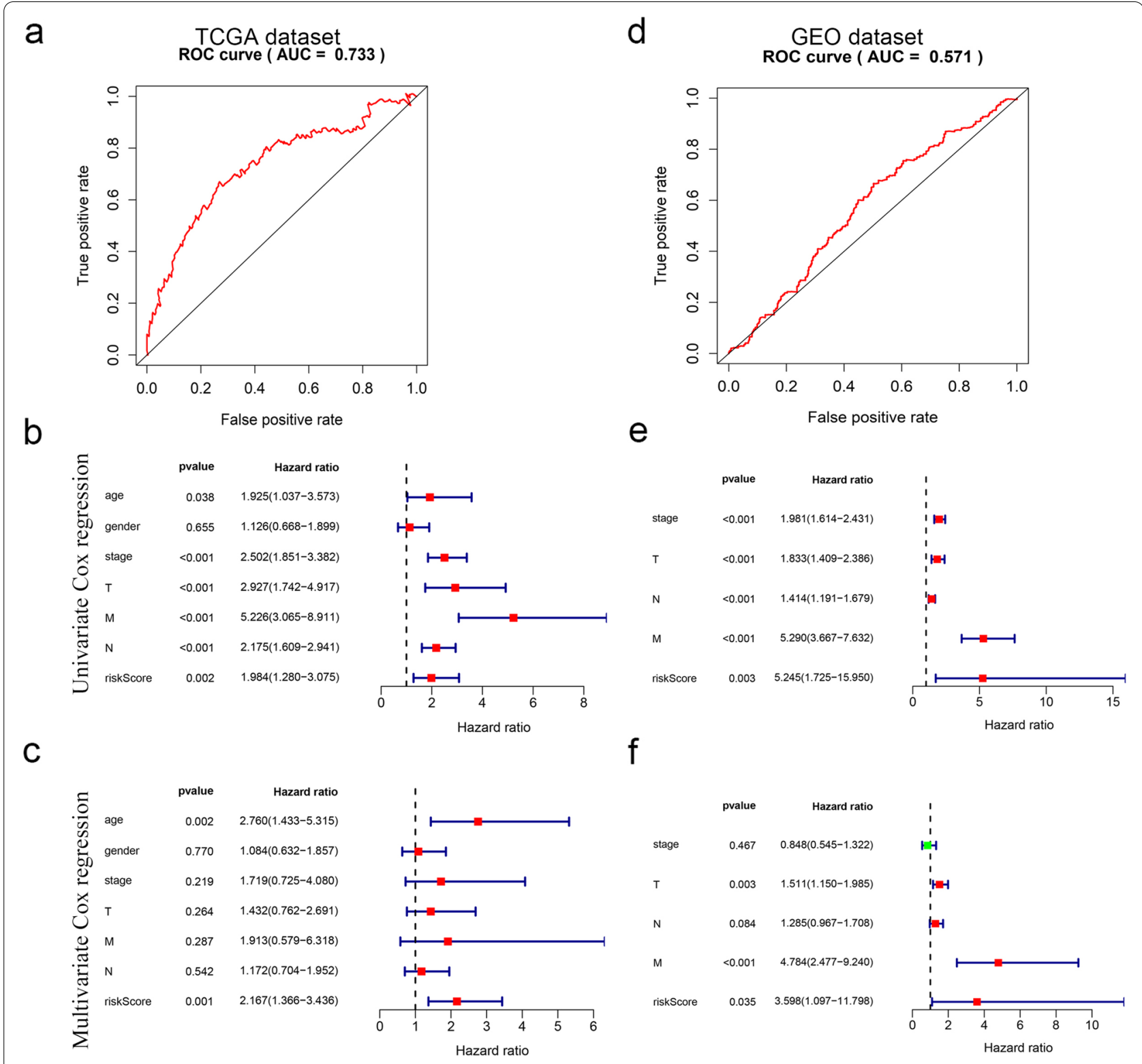

Fig. 8 Prognostic value of two-gene signature in two datasets. a ROC curves, $\mathbf{b} \cup C R$, and $\mathbf{c} M C R$ analysis of the risk score and other clinical indices in TCGA cohort. $\mathbf{d}$ ROC curves, e UCR, and $\mathbf{f} M C R$, analysis of the risk score and other clinical indices in GEO cohort

PPARGC1A expression in tumor immunity. This needs further research in the future.

Next, the risk score was employed to divide patients into low- and high-risk group. K-M curve, score plot, and survival status plot, as well as ROC curve demonstrated that the two IARBPs had a favorable estimation potential in TCGA and GEO databases.

Furthermore, we researched on the relation of the two immune-associated RBPs prognostic signature to immune microenvironment. The high-risk group had significantly higher ESTIMATE score and immune score compared to low-risk group. For the type of infiltrating immune cells, the degree of infiltration of Macrophages M0 and Macrophages M2 were significantly upregulated. In the immune microenvironment of $\mathrm{CC}$, tumor-associated macrophages (TAMs) are one of major tumor-infiltrating immune cells [35]. Although macrophages should be able to kill tumor cells, immunosuppressive microenvironment most often polarizes TAMs into M2-like macrophages rather than M1-like macrophages, which promote immunosuppression, angiogenesis, and extracellular matrix [36]. Many studies 


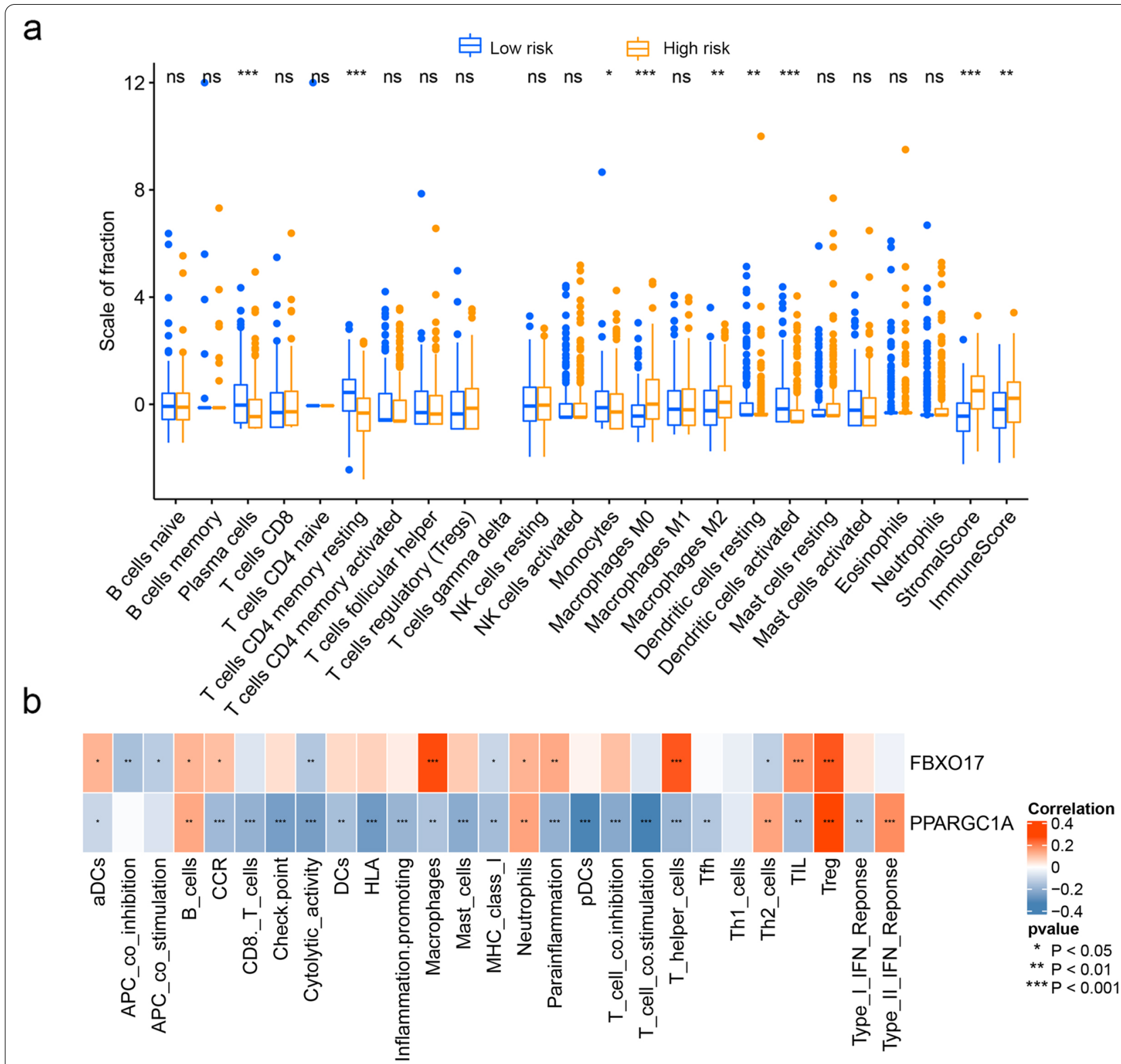

Fig. 9 Analysis of the immune gene sets between different risk groups in the TCGA cohort. a The immune infiltration in high- and low-risk patients. b Correlation heatmap of two RBPs (FBXO17 and PPARGC1A) and immune gene sets. ${ }^{*} P<0.05,{ }^{* *} P<0.01,{ }^{* *} P<0.005$

have reported that the presence of large numbers of M2 macrophages in colorectal carcinoma is significantly correlated with decreased survival rates [37, 38]. M2 macrophage infiltration is positively related with the expression of immune checkpoints. Furthermore, antiM2 macrophages combined with immune checkpoint inhibitors improves the therapeutic effect and provides a new idea for the treatment of tumors [39]. Contrary to the pro-inflammatory function during infections, tumor-associated neutrophils (TANs) promotes tumor progression malignancy by mediating angiogenesis [40].
This indicated that high-risk patients might be in an immunosuppressive state and have a poor effect to the immunotherapy.

Furthermore, the CellMiner database was exploited to analyze the relationship between the drug sensitivity and the expression of FBXO17 and PPARGC1A. The result indicated that FBXO17 was most sensitive to treatment with Molecular targeted therapy drugs such as Irofulven, Sonidegib, and Lenvatinib. In addition, the PPARGC1A was most sensitive to Vemurafenib and Dabrafenib. 


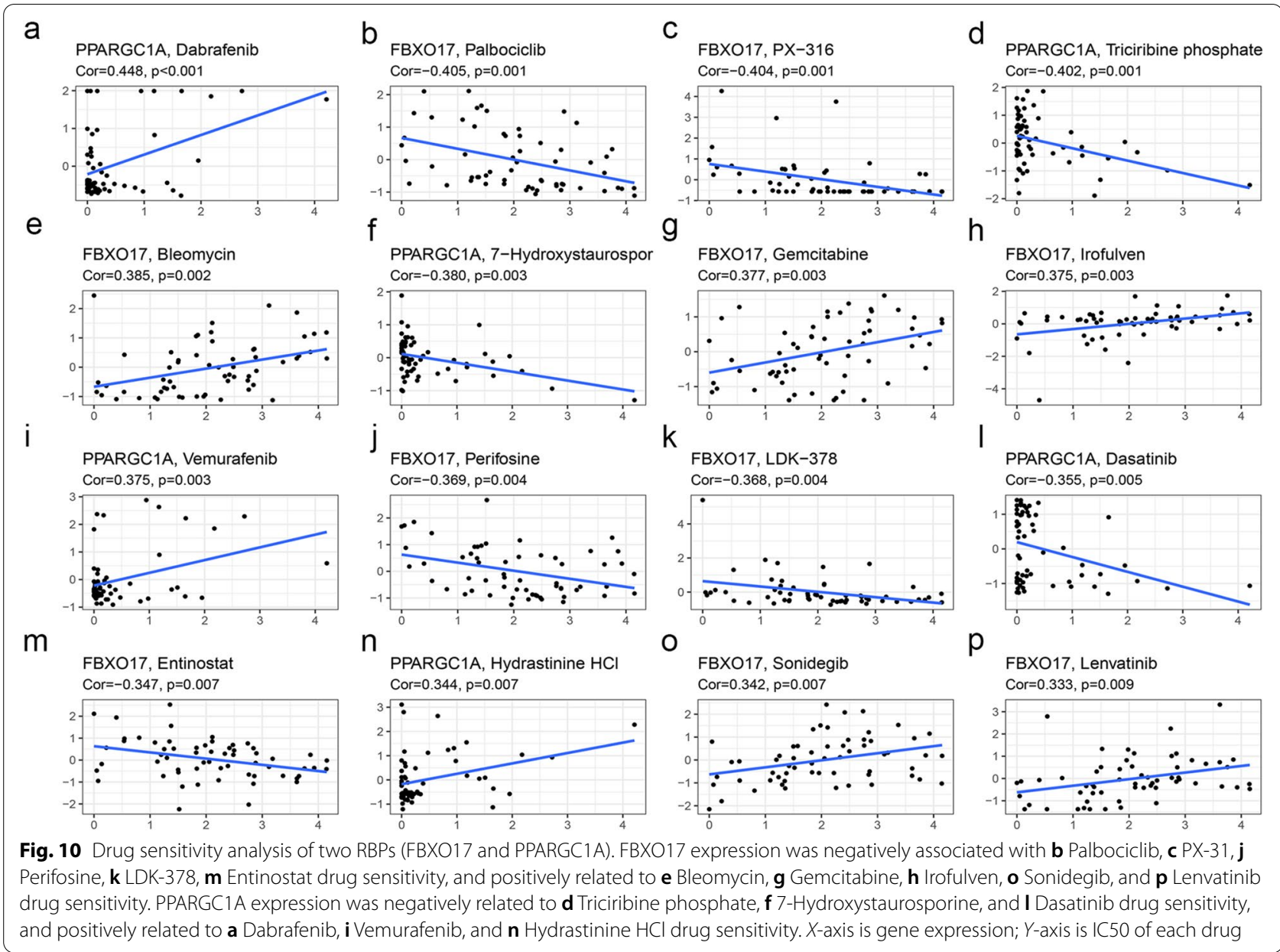

In conclusion, we performed a comprehensive bioinformatics analysis of RBPs and identified a novel prognostic risk score involving two RBPs in CC. This risk model can be used as a biomarker to independently predict the prognosis of $\mathrm{CC}$ and may offer new promising therapeutic targets for CC patients. The limitation of this study is that further research is still needed to verify our findings.

\section{Abbreviations}

TCGA: The Cancer Genome Atlas databases; GEO: Gene Expression Omnibus; CC: Colon cancer; WGCNA: Co-expression network analysis; RBPs: RNA-binding proteins; IARBPs: Immune-associated RNA-binding proteins; DEGs: Differentially expressed genes; GO: Gene Ontology; UCR: Univariate Cox regression; MCR: Multivariate Cox regression; ssGSEA: Single sample gene set enrichment analysis.

\section{Supplementary Information}

The online version contains supplementary material available at https://doi. org/10.1186/s12957-021-02411-2.

Additional file 1: Figure S1. The work-flow of this study.

\section{Acknowledgements}

None.

\section{Authors' contributions}

DS and KY contributed to the conception and design of the study. DS collected data and wrote the manuscript. DS performed the data analysis and constructed the figures and tables. KY and JC reviewed and revised the manuscript and were involved in the conception of the study. Additionally, ZW was responsible for the organization, revision, and submission of this manuscript. The authors read and approved the final manuscript.

\section{Funding}

None.

\section{Availability of data and materials}

The datasets analyzed were acquired from The Cancer Genome Atlas (TCGA) database (https://portal.gdc.cancer.gov/) and GEO database (http://www.ncbi. nlm.nih.gov/geo/).

\section{Declarations}

\section{Ethics approval and consent to participate}

This was not applicable to this manuscript.

\section{Consent for publication}

Consent for publication was obtained from all participants. 


\section{Competing interests}

The authors declare that they have no competing interests.

\section{Author details}

${ }^{1}$ Department of Gastrointestinal Surgery, Affiliated Hospital of Yangzhou University, Yangzhou 225100, People's Republic of China. ${ }^{2}$ Department of General Surgery, People's Hospital of Jingjiang, Yangzhou University Medical Academy, Yangzhou, China.

Received: 4 June 2021 Accepted: 29 September 2021

Published online: 26 October 2021

\section{References}

1. Sung H, Ferlay J, Siegel R, Laversanne M, Soerjomataram I, Jemal A, et al. Global Cancer Statistics 2020: GLOBOCAN estimates of incidence and mortality worldwide for 36 cancers in 185 countries. CA Cancer J Clin. 2021;71:209-49.

2. Brenner $\mathrm{H}$, Chen $\mathrm{C}$. The colorectal cancer epidemic: challenges and opportunities for primary, secondary and tertiary prevention. Br J Cancer. 2018;119:785-92.

3. Huang F, Peng Y, Ye Q, Chen J, Li Y, Liu S, et al. CILP2 overexpression correlates with tumor progression and poor prognosis in patients with colorectal cancer in The Cancer Genome Atlas (TCGA) study. World J Surg Oncol. 2020;18:274

4. Li WX, Zheng JJ, Zhao G, Lyu CT, Lu WQ. Overexpression of DSCR1 prevents proliferation and predicts favorable prognosis in colorectal cancer patients. World J Surg Oncol. 2021;19:100.

5. Bhandari A, Woodhouse M, Gupta S. Colorectal cancer is a leading cause of cancer incidence and mortality among adults younger than 50 years in the USA: a SEER-based analysis with comparison to other young-onset cancers. J Investig Med. 2017;65:311-5.

6. Xu L, Zheng Q. Identification and validation of a miRNA-related expression signature for tumor mutational burden in colorectal cancer. World J Surg Oncol. 2021;19:56

7. Fang Z, Xu S, Xie Y, Yan W. Identification of a prognostic gene signature of colon cancer using integrated bioinformatics analysis. World J Surg Oncol. 2021:19:13.

8. Cai R, Lu Q, Wang D. Construction and prognostic analysis of miRNA-mRNA regulatory network in liver metastasis from colorectal cancer. World J Surg Oncol. 2021;19(7)

9. Wang $X, X u$ Y, Li T, Chen B, Yang W. Development of prognosis model for colon cancer based on autophagy-related genes. World J Surg Oncol. 2020;18:285.

10. Qu Y, Chen Y, Zhang L, Tian L. Construction of prognostic predictor by comprehensive analyzing alternative splicing events for colon adenocarcinoma. World J Surg Oncol. 2020;18:236.

11. Binnewies M, Roberts EW, Kersten K, Chan V, Fearon DF, Merad M, et al. Understanding the tumor immune microenvironment (TIME) for effective therapy. Nat Med. 2018;24:541-50.

12. Gajewski TF, Schreiber H, Fu YX. Innate and adaptive immune cells in the tumor microenvironment. Nat Immunol. 2013;14:1014-22.

13. Wurth $L$, Gebauer F. RNA-binding proteins, multifaceted translational regulators in cancer. Biochim Biophys Acta. 1849;2015:881-6.

14. Dreyfuss G, Kim V, Kataoka N. Messenger-RNA-binding proteins and the messages they carry. Nat Rev Mol Cell Biol. 2002;3:195-205.

15. Turner M, Díaz-Muñoz M. RNA-binding proteins control gene expression and cell fate in the immune system. Nat Immunol. 2018;19:120-9.

16. Gerstberger $S$, Hafner $M$, Tuschl T. A census of human RNA-binding proteins. Nat Rev Genet. 2014;15:829-45.

17. Yoshihara K, Shahmoradgoli M, Martínez E, Vegesna R, Kim H, TorresGarcia W, et al. Inferring tumour purity and stromal and immune cell admixture from expression data. Nat Commun. 2013:4:2612.

18. Newman A, Liu C, Green M, Gentles A, Feng W, Xu Y, et al. Robust enumeration of cell subsets from tissue expression profiles. Nat Methods. 2015;12:453-7.

19. Langfelder P, Horvath S. WGCNA: an R package for weighted correlation network analysis. BMC Bioinformatics. 2008;9:559.
20. Shankavaram U, Varma S, Kane D, Sunshine M, Chary K, Reinhold W, et al. CellMiner: a relational database and query tool for the $\mathrm{NCl}-60$ cancer cell lines. BMC Genomics. 2009:10:277.

21. Reinhold W, Sunshine M, Liu H, Varma S, Kohn K, Morris J, et al. CellMiner: a web-based suite of genomic and pharmacologic tools to explore transcript and drug patterns in the $\mathrm{NCl}-60$ cell line set. Eur J Cancer. 2012;72:3499-511.

22. Dekker E, Tanis PJ, Vleugels JLA, Kasi PM, Wallace MB. Colorectal cancer. Lancet. 2019:394:1467-80.

23. Mohsen N, Reza M. The global, regional, and national burden of colorectal cancer and its attributable risk factors in 195 countries and territories, 1990-2017: a systematic analysis for the Global Burden of Disease Study 2017. Lancet Gastroenterol Hepatol. 2019;4:913-33.

24. Finotello F, Rieder D, Hackl H, Trajanoski Z. Next-generation computational tools for interrogating cancer immunity. Nat Rev Genet. 2019;20:724-46.

25. Helmy KY, Patel SA, Nahas GR, Rameshwar P. Cancer immunotherapy: accomplishments to date and future promise. Ther Deliv. 2013;4:1307-20.

26. Yang Y. Cancer immunotherapy: harnessing the immune system to battle cancer. J Clin Invest. 2015;125:3335-7.

27. Hurtado C, Wan F, Housseau F, Sears C. Roles for interleukin 17 and adaptive immunity in pathogenesis of colorectal cancer. Gastroenterology. 2018;155:1706-15

28. Lunde BM, Moore C, Varani G. RNA-binding proteins: modular design for efficient function. Nat Rev Mol Cell Biol. 2007;8:479-90.

29. Liu FH, Cui YP, He YK, Shu RH. FBXO17 promotes malignant progression of hepatocellular carcinoma by activating wnt/ $\beta$-catenin pathway. Eur Rev Med Pharmacol Sci. 2019;23:8265-73.

30. Suber TL, Nikolli I, O'Brien ME, Londino J, Zhao J, Chen K, et al. FBXO17 promotes cell proliferation through activation of Akt in lung adenocarcinoma cells. Respir Res. 2018;19:206.

31. Peng D, Wang Z, Huang A, Zhao Y, Qin FX. A novel function of F-box protein $\mathrm{FBXO} 17$ in negative regulation of type I IFN signaling by recruiting PP2A for IFN regulatory factor 3 deactivation. J Immunol. 2017;198:808-19.

32. Cantó C, Auwerx J. PGC-1??, SIRT1 and AMPK, an energy sensing network that controls energy expenditure. Curr Opin Lipidol. 2009;20:98-105.

33. Cho YA, Lee J, Oh JH, Chang HJ, Sohn DK, Shin A, et al. Genetic variation in PPARGC1A may affect the role of diet-associated inflammation in colorectal carcinogenesis. Oncotarget. 2017;8:8550-8.

34. Handschin C, Spiegelman BM. The role of exercise and PGC1alpha in inflammation and chronic disease. Nature. 2008;454:463-9.

35. Wei C, Yang C, Wang S, Shi D, Zhang C, Lin X, et al. Crosstalk between cancer cells and tumor associated macrophages is required for mesenchymal circulating tumor cell-mediated colorectal cancer metastasis. Mol Cancer. 2019;18.

36. Becht E, Giraldo NA, Germain C, Reyniès A, Fridman W. Immune contexture, immunoscore, and malignant cell molecular subgroups for prognostic and theranostic classifications of cancers. Adv Immunol. 2016:130:95-190

37. Xue T, Yan K, Cai Y, Sun J, Chen Z, Chen X, et al. Prognostic significance of CD163+ tumor-associated macrophages in colorectal cancer. World J Surg Oncol. 2021;19:186

38. Wang Y, Wang J, Yang C, Wang Y, Liu J, Shi Z, et al. A study of the correlation between $\mathrm{M} 2$ macrophages and lymph node metastasis of colorectal carcinoma. World J Surg Oncol. 2021;19:91.

39. Ju X, Zhang H, Zhou Z, Chen M, Wang Q. Tumor-associated macrophages induce PD-L1 expression in gastric cancer cells through IL-6 and TNF-a signaling. Exp Cell Res. 2020;396:112315.

40. Fujita M, Scheurer ME, Decker SA, Mcdonald HA, Kohanbash G, Kastenhuber ER, Kato H, Bondy ML, Ohlfest JR, Okada HJ. Role of type 1 IFNs in antiglioma immunosurveillance--using mouse studies to guide examination of novel prognostic markers in humans. Clin Cancer Res. 2010;16:3409-19.

\section{Publisher's Note}

Springer Nature remains neutral with regard to jurisdictional claims in published maps and institutional affiliations. 